\title{
ORIGINAL
}

\section{METODOLOGÍA EMPLEADA EN LOS ARTÍCULOS ORIGINALES PUBLICADOS SOBRE TABAQUISMO EN CUATRO REVISTAS MÉDICAS ESPAÑOLAS (1985-1996)}

\author{
José Antonio García López
}

Departamento de Farmacia y Tecnologia Farmacéutica. Universidad de Granada.

\section{RESUMEN}

Fundamento: Sicndo el discño de una investigación el factor más importante de la calidad de los resultados obtenidos y el tabaquismo un tema prioritario de investigación, se describen las características metodológicas de los artículos originales sobre tabaquismo publicados en cuatro revistas médicas españolas.

Métodos: Se realizó un estudio descriptivo de los artículos originales sobre tabaquismo, procedentes de centros españoles, publicados en el periodo de 1985 a 1996 en las revistas Atención Primaria, Medicina Clínica (Barcelona), Revista Española de Salud Pública y Revista Clínica Española.

Resultados: De los 154 originales analizados, la mayoría trataron los temas de patrones de consumo $(31,8 \%)$ y del tabaquismo como factor de riesgo $(27,3 \%)$, se realizaron a nivel municipal $(41,7 \%)$ o provincial $(36,1 \%)$, con sujetos procedentes de la atención primaria $(71,6 \%)$, que acudían a consulta $(20,8 \%)$, con edades comprendidas entre 25 y 65 años $(30,4 \%)$ o mayores de 15 años $(27,0 \%)$. Se emplearon encuestas para obtener la información $(38,3 \%$ ) y tuvieron una duración de 12 meses $(24,5 \%)$ o más $(19,3 \%)$. Los diseños más utilizados fueron descriptivos $(67,5 \%)$, retrospectivos $(79,2 \%)$ y transversales $(81,8 \%)$.

Conclusiones: La investigación española sobre tabaquismo debe cubrir ciertos aspectos temáticos no tratados suficientemente y cuidar ciertos aspectos metodológicos que mejoren la calidad de las publicaciones, necesidad apuntada también en otros estudios.

Palabras clave: Atención Primaria. Diseño. España. Investigación. Medicina Clínica (Barcelona). Revistas biomédicas. Revista Clinica Española. Revista Española de Salud Pública. Tabaquismo.

Correspondencia:

José Antonio García López

Departamento de Farmacia y Tecnología Farmacéutica.

Facultad de Farmacia.

Universidad de Granada.

Campus de la Cartuja, s/n.

Correo clcctrónico: jagarcia@platon.ugr.es.

\section{ABSTRACT \\ Methodology employed in the original articles published on the cigarette smoking habit in four Spanish medical journals (1985-1996)}

Background: Planning being the one factor entailed in doing any research that has the greatest bearing on the quality of the findings thereof, and the cigarette smoking habit being a prime topic for research, a description is provided of the methodological aspects of the original articles on the cigarette smoking habit which have been published in four Spanish medical journals.

Methods: A descriptive study was conducted regarding the original articles on the cigarette smoking habit from Spanish centers which were published in 1985-1996 in the journals Atención Primaria (Primary Care), Medicina Clinica (Clinical Medicine) (Barcelona), Revista Española de Salud Pública (Spanish Public Health Journal) and Revista Clinica Española (Spanish Clinical Journal).

Results: Most of the 154 original articles studied dealt with the topics of smoking patterns $(31.8 \%)$ and the cigarette smoking habil as a risk factor $(27.3 \%)$ and were conducted within a municipal scope $(41.7 \%)$ or provincial scope (36.1\%) with subjects from primary care $(71.6 \%)$ who visited the doctor $(20.8 \%)$ falling within the age $25-65$ group $(30.4 \%)$ or over age $15(27.0 \%)$. Surveys were used for collecting the data $(38.3 \%)$ and were conducted throughout a 12 -month period $(24.5 \%)$ or longer $(19.3 \%)$. The types most frequently used were descriptive $(67.5 \%)$, retrospective $(79.2 \%)$ and cross-sectional $(81.8 \%)$.

Conclusions: Spanish research on the cigarette smoking habit must cover certain subjects which have not been dealt with in sufficient depth and must invest greater care regarding certain methodological aspects which will improve the quality of the publications, this being a need also brought to fore in other studies.

Key words: Atención Primaria. Medicina Clínica (Barcelona). Health Journals. Revista Clínica Española. Revista Española de Salud Pública. Research. Spain. Tobacco. 


\section{INTRODUCCIÓN}

Los artículos publicados en las revistas científicas constituyen el medio fundamental de comunicación entre la comunidad científica de biomedicina, permitiendo a los profesionales mantener y actualizar sus conocimientos. Las referencias a los artículos de revistas alcanzan en medicina en general el $92,1 \%$ y en inmunología en particular, el $94,3 \% 1$. Fl estudio de los artículos publicados en revistas científicas ofrece una posibilidad de aproximación a la situación real de la investigación que se está realizando, bien sea por instituciones, áreas geográficas, profesionales, revistas o áreas temáticas.

Los artículos deben utilizar una metodología de trabajo adecuada que asegure la validez de los resultados y deben contener la información suficiente para poder evaluarlos críticamente. El diseño de un trabajo es el factor más importante de la calidad de los resultados obtenidos ${ }^{2}$.

En España se han publicado, en los últimos años, artículos científicos que se preocupan por algunos aspectos metodológicos de la investigación, como el tratamiento estadístico de los datos ${ }^{3-5} \mathrm{o}$ los tipos de diseño empleados $^{2,6,7}$, generalmente relativos a una o varias revistas cientificas ${ }^{8-10}$ o a una determinada especialidad ${ }^{11-13}$, no habiéndose abordado el problema de la metodología relativa a temas de investigación.

Siendo el tabaquismo el primer problema sanitario susceptible de prevención, la mayoría de los países desarrollados han puesto en marcha planes de lucha antitabáquica, siguiendo las recomendaciones de la $\mathrm{OMS}^{14} \mathrm{y}$ han fomentado la investigación en este campo, considerándolo como un tema prioritario. En España, el plan de Salud para Todos en el año 2000 incluye cuatro grandes estrategias, siendo la primera la «Promoción de hábitos saludables de vida», cuyo primer apartado, de los siete que contempla, es el tabaco ${ }^{15}$. A pesar de la importancia de la investigación sobre el tabaquismo no se han publicado trabajos que estudien las características metodológicas de la misma, tanto a nivel nacional como internacional.

El objetivo de este trabajo es describir algunos aspectos metodológicos utilizados en los artículos originales sobre tabaquismo publicados en cuatro revistas españolas, para obtener una aproximación a la realidad de la investigación española sobre tabaquismo.

\section{MATERIAL Y MÉTODOS}

Se eligieron las revistas Atención Primaria (Aten Primaria), Medicina Clínica (Barcelona) (Med Clin [Barc]), Revista Española de Salud Pública (Rev Esp Salud Pública) y Revista Clínica Española (Rev Clin Esp) por ser, de acuerdo con la base de datos del Indice Médico Español, las que más artículos originales sobre tabaquismo tenían publicados.

De cada revista se seleccionaron manualmente todos los artículos relacionados con el tabaquismo incluidos en la sección de originales, publicados entre 1985 y 1996, y realizados en centros españoles. Se obtuvieron 154 originales de cuyo examen, particularmente de la sección «Material y métodos», se extrajeron las siguientes variables: revista, año de publicación, tema de investigación, financiación, ámbito geográfico y asistencial, procedencia y edad de los sujetos del estudio, fuente de obtención de los datos, duración del estudio y diseño según la manipulación, comienzo y seguimiento.

La evaluación de dichas variables se efectuó por un solo observador. Se realizó un estudio descriptivo de carácter retrospectivo. Las variables relacionadas con el diseño se estudiaron longitudinalmente, dividiendo el período de ticmpo considerado en cuatro trienios. El resto de las variables se estudiaron transversalmente.

Para identificar el tema de investigación, sólo a modo de orientación, se utilizaron los 
descriptores del Tesauro de Drogodependencias $^{16}$, asignándosele a cada artículo una sola categoría. El diseño de los estudios se estableció de acuerdo con la clasificación de Gálvez y $\operatorname{col}^{17}$, cuyas ventajas han sido discutidas por algunos autores ${ }^{18}$. Según la manipulación los estudios se clasificaron en observacionales y experimentales. Los primeros se clasificaron en descriptivos y analíticos. Los estudios descriptivos incluyen series de casos, estudios descriptivos de mortalidad y morbilidad, correlaciones temporales, correlaciones ecológicas, estudios de mortalidad proporcional y estudios de prevalencia. Los estudios analíticos incluyen estudios de casos y controles y de cohortes. Los estudios experimentales incluyen ensayos comunitarios y ensayos clinicos. Según el momento del estudio se clasificaron en retrospectivos, prospectivos y ambispectivos. Según el seguimiento los estudios se clasificaron en longitudinales y transversales.
Los datos se procesaron en un ordenador personal, utilizando el programa Statgraphics 7.0 y se expresaron como frecuencias absolutas y relativas. Se utilizó la prueba de la chi cuadrado para la comparación de proporciones. El nivel de significación se fijó al 5\%.

\section{RESULTADOS}

En el período de 1985 a 1996, las cuatro revistas objeto de estudio publicaron 3.955 artículos originales, de los que $154(3,9 \%)$ correspondían a tabaquismo. De estos, 59 $(38,3 \%)$ se publicaron en Aten Primaria, 52 (33,8\%) en Med Clin (Barc), 25 (16,2\%) en Rev Esp Salud Pública y $18(11,7 \%)$ en Rev Clin Esp.

Los aspectos temáticos más frecuentemente tratados (figura 1) fueron los patrones de consumo $(31,8 \%)$, que incluían estudios sobre

Figura 1

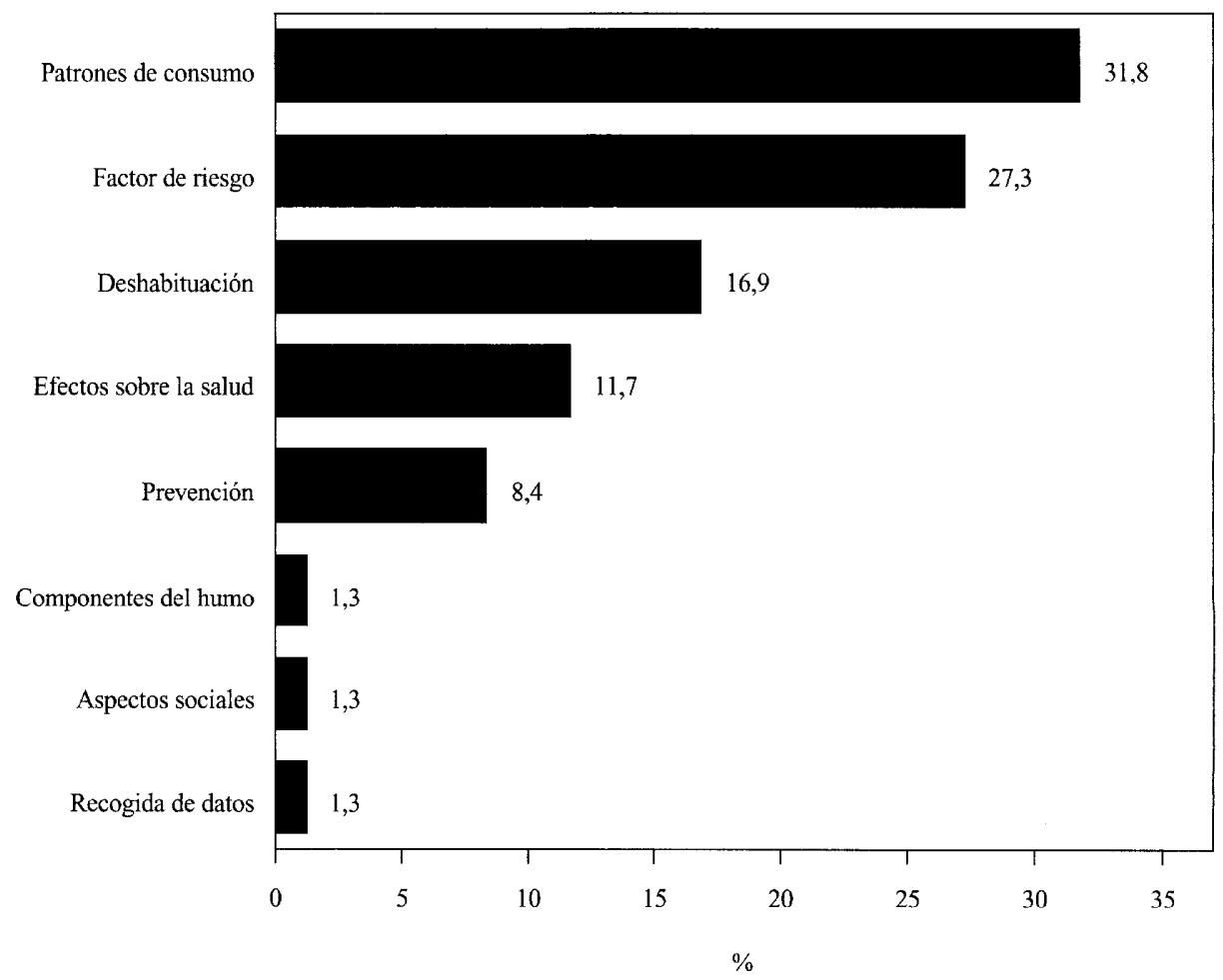


hábitos, prevalencia, actitudes, conocimicntos y conductas frente al tabaquismo; y el tabaquismo como factor de riesgo $(27,3 \%)$.

Sólo $24(15,6 \%)$ originales indicaban la financiación, de los que $14(58,3 \%)$ fueron financiados por el Fondo de Investigaciones Sanitarias y $10(41,7 \%)$ por otras tantas instituciones.

El ámbito geográfico no sc indicaba en 10 $(6,5 \%)$ originales, y en el resto predominaron el ámbito municipal $(41,7 \%)$ y el provincial $(36,1 \%)$ (figura 2 ). El ámbito asis- tencial no se indicaba en $6(3,9 \%)$ originales. En el resto, predominaron la atención primaria $(71,6 \%)$ y la asistencia hospitalaria $(21,6 \%)$. Los dos ámbitos estuvicron presentes en $10(6,8 \%)$ originales.

Los sujetos que con mayor frecuencia formaban parte del estudio (figura 3 ) fueron los que acudían a consulta $(20,8 \%)$. La edad de los mismos no figuraba en $6(3,9 \%)$ originales. Los grupos de edad más frecuentes (tabla 1) fueron los de 26-65 años $(30,4 \%)$ y los mayores de 15 años $(27,0 \%)$.

Figura 2

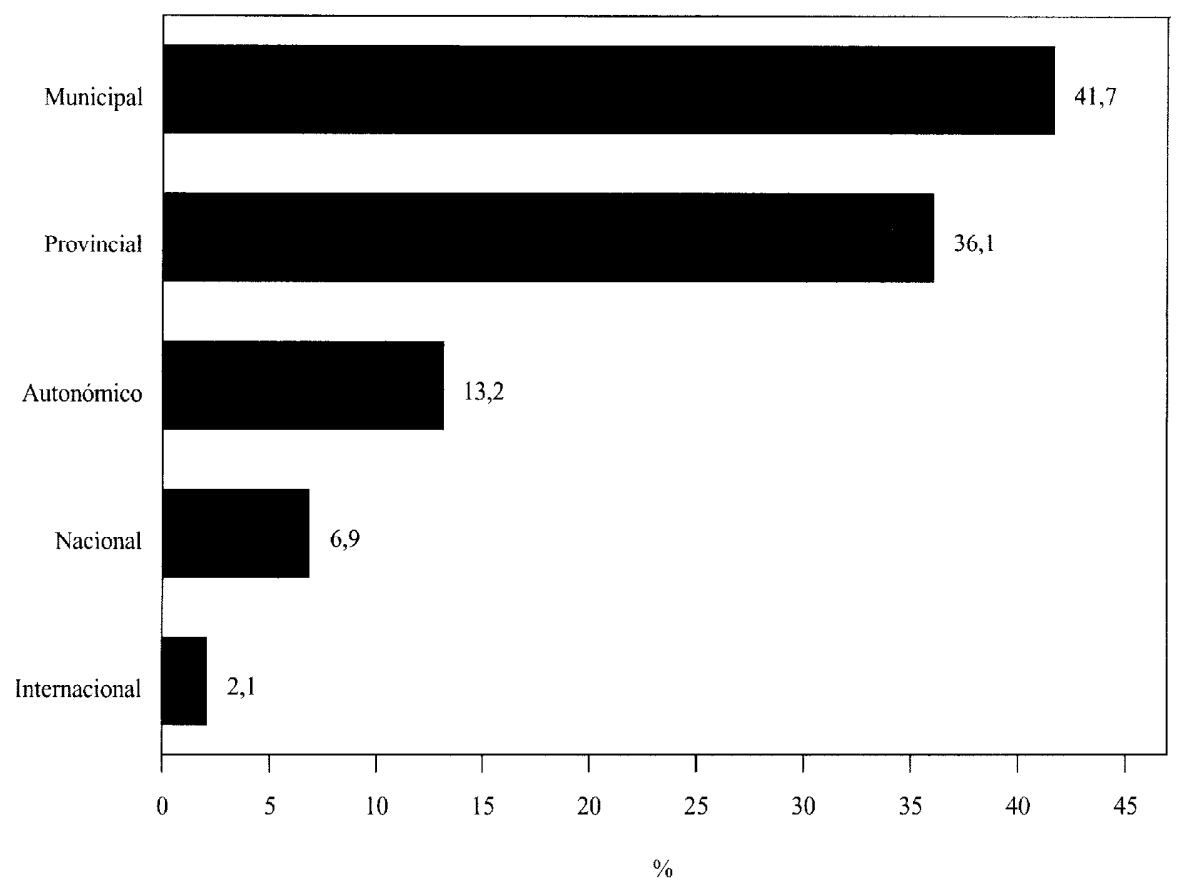

Las encuestas fueron la fuente de información más utilizada para llevar a cabo los estudios (tabla 2$)$, bien sola $(38,3 \%)$ o asociada al examen de salud $(27,3 \%)$ o a las historias clínicas $(2,6 \%)$.

Los períodos de tiempo que incluían los estudios no se indicaban en $40(26,0 \%)$ originales. En el resto, predominaron los ar- tículos con una duración de 12 meses $(24,5 \%)$ y de más de 12 meses $(19,3 \%)$ (tabla 3 ).

Según la manipulación, el 67,5\% de los estudios fucron descriptivos (figura 4). No sc detectaron diferencias significativas en los cuatro trienios en que se agruparon los 12 años que abarcó el estudio $\left(\chi^{2}=5,8608 ; \mathrm{gl}=6\right.$; 


\section{Figura 3}

Sujetos que acuden a consulta

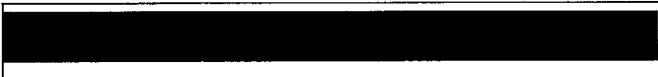

20,8

Población general

酯

Pacientes con accidente vascular

Profesionales sanitarios

Voluntarios fumadores

Trabajadores de empresa

Pacientes con enfermedad crónica

Pacientes con cáncer

Pacientes con úlcera

Otros sujetos

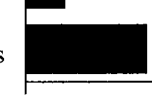

0

Tabla 1

Número de originales(\%) publicados sobre tabaquismo según la edad de los sujetos objeto de estudio

\begin{tabular}{|lc|}
\hline Edad (años) & $N(\%)$ \\
\hline$<11$ & $1(0,7)$ \\
$>7$ & $3(2,0)$ \\
$6-16$ & $3(2,0)$ \\
$11-15$ & $7(4,7)$ \\
$11-25$ & $1(0,7)$ \\
$16-20$ & $3(2,0)$ \\
$15-25$ & $2(1,4)$ \\
$>15$ & $40(27,0)$ \\
$18-32$ & $1(0,7)$ \\
$>19$ & $9(6,1)$ \\
$21-25$ & $2(1,4)$ \\
$20-64$ & $6(4,1)$ \\
$22-40$ & $4(2,6)$ \\
$>24$ & $2(1,4)$ \\
$26-65$ & $45(30,4)$ \\
$>30$ & $6(4,1)$ \\
$>40$ & $2(1,4)$ \\
$>44$ & $3(2,0)$ \\
$>50$ & $1(0,7)$ \\
$>65$ & $4(2,6)$ \\
Todas las edades & $3(2,0)$ \\
\hline Total & $148(100)$ \\
\hline
\end{tabular}

Tabla 2

12,4

11

11

11

10,4

9,8

5,2

3,2

3,9

5

10

$\%$

15

20

25

Número de originales (\%) publicados sobre tabaquismo según la fuente de obtención de los datos

\begin{tabular}{|lc|}
\hline Fuente de obtención de datos & $N(\%)$ \\
\hline Encuestas & $59(38,3)$ \\
Encuestas y examen de salud & $42(27,3)$ \\
Examen de salud & $18(11,7)$ \\
Historias clínicas & $18(11,7)$ \\
Historias clínicas y examen de salud & $6(3,9)$ \\
Bibliografia & $7(4,5)$ \\
Encuestas e historias clínicas & $4(2,6)$ \\
\hline Total & $154(100)$ \\
\hline
\end{tabular}

Tabla 3

Número de originales $(\%)$ publicados sobre tabaquismo según la duración del estudio

\begin{tabular}{|lc|}
\hline Duración (meses) & $N(\%)$ \\
\hline 1 & $14(12,3)$ \\
2 & $13(11,4)$ \\
3 & $8(7,0)$ \\
4 & $6(5,3)$ \\
5 & $2(1,8)$ \\
6 & $11(9,6)$
\end{tabular}




\begin{tabular}{|lc|}
\hline Duración (meses) & $N(\%)$ \\
\hline 7 & $1(0,9)$ \\
8 & $3(2,6)$ \\
9 & $3(2,6)$ \\
10 & $2(1,8)$ \\
11 & $1(0,9)$ \\
12 & $28(24,5)$ \\
Más de 12 & $22(19,3)$ \\
\hline Total & $114(100)$ \\
\hline
\end{tabular}

$\mathrm{p}=0,4389$ ). En función de la revista de publicación (tabla 4) se encontraron diferencias significativas $\left(\chi^{2}=11,1746 ; \mathrm{gl}=3 ; \mathrm{p}=0,0108\right)$, de forma que en Aten Primaria y en Rev Esp Salud Pública los porcentajes de estudios descriptivos fueron mayores que en $\mathrm{Med}$ Clin (Barc) y en Rev Clin Esp. En el cálculo del estadígrafo se agruparon las categorías de estudios analíticos y experimentales, para que el test cumpliera las condiciones de validez.

Figura 4

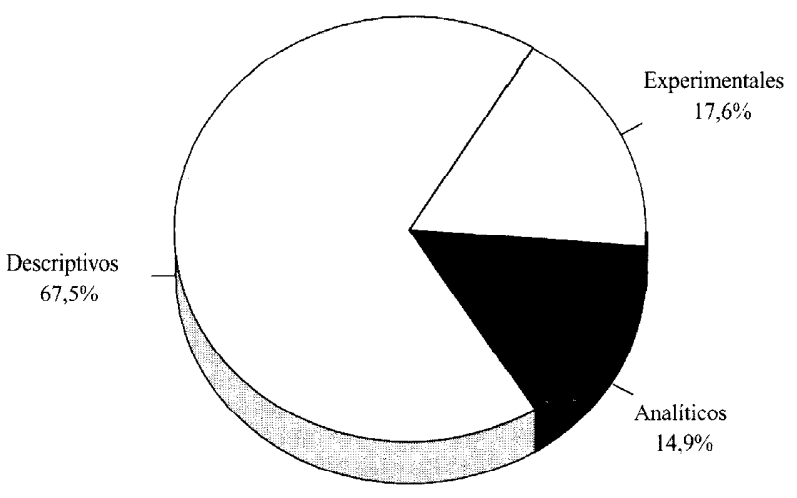

Tabla 4

Número de originales (\%) publicados sobre tabaquismo según la manipulación de los estudios y revista de publicación

\begin{tabular}{|lcccc|}
\hline Estudios & Med. Clin. (Barc) & Aten. Primaria & $\begin{array}{c}\text { Rev. Esp. Salud } \\
\text { Pública }\end{array}$ & Rev. Clin. Esp. \\
\hline Descriptivos & $29(55,8)$ & $48(81,4)$ & $18(72,0)$ & $9(50,0)$ \\
Analíticos & $15(28,8)$ & $2(3,4)$ & $2(8,0)$ & $4(22,2)$ \\
Experimentales & $8(15,4)$ & $9(15,3)$ & $5(20,0)$ & $5(27,8)$ \\
\hline Total & $52(33,8)$ & $59(38,3)$ & $25(16,2)$ & $18(11,7)$ \\
\hline
\end{tabular}

Según el momento del estudio, 122 (79,2\%) originales eran estudios retrospectivos, 30 $(19,5 \%)$ prospectivos y $2(1,3 \%)$ ambispectivos. No se encontraron diferencias significativas entre los cuatro trienios $\left(\chi^{2}=3,1160\right.$; $\mathrm{gl}=3 ; \mathrm{p}=0,3740)$ ni entre las cuatro revistas $\left(\chi^{2}=6,3640 ; g l=3 ; p=0,0951\right)$. En ambos casos hubo que agrupar las categorías de estu- dios prospectivos y ambispectivos para que el test estadístico cumpliera las condiciones de validez.

Según el seguimiento, $126(81,8 \%)$ originales incluían estudios transversales y 28 $(18,2 \%)$ longitudinales. No se encontraron diferencias significativas entre los cuatro 
trienios $\left(\chi^{2}=0,4904 ; \mathrm{g}=3 ; \mathrm{p}=0,9209\right)$ ni entre las cuatro revistas $\left(\chi^{2}=1,2087 ; \mathrm{gl}=3\right.$; $\mathrm{p}=0,7509$ ).

\section{DISCUSIÓN}

Las revistas seleccionadas en el presente estudio, además de ser las que más trabajos originales publican sobre tabaquismo, tienen reconocido prestigio, ya que aparecen indexadas en la mayoría de las bases de datos internacionales ${ }^{19}$ y son las más citadas en la literatura médica española ${ }^{20}$. Otras revistas que no posean estos distintivos, presumiblemente contendrán diseños metodológicos menos complejos de los aquí encontrados. La evaluación de los aspectos metodológicos se realizó por un único observador, lo que puede constituir otra limitación de este trabajo.

Consideramos que las características metodológicas analizadas fueron suficientemente informadas en los originales sobre tabaquismo, con la excepción de la duración de los estudios, de la que no se informó en el $26,0 \%$ de los originales. Esta situación es más favorable que la encontrada por Jiménez y $\mathrm{col}^{9}$ analizando 18 características metodológicas en los originales publicados en Aten Primaria en 1990, en los que se comprueba que 6 características son informadas insuficientemente en el $45 \%$ de los artículos.

El alto porcentaje de artículos que no indicaron la financiación ha sido encontrado también en 446 originales de 34 revistas del ámbito de la atención primaria en el período de $1988-1992^{10}$, pudiéndose atribuir a que no se especificaba la misma o a que se habían realizado con una subvención indirecta al efectuarse durante la jornada laboral y con el material disponible en los centros de trabajo. Las revistas deberían vigilar la declaración efectiva de este aspecto tan importante, a fin de evitar posibles conflictos de intereses.
Los ámbitos geográficos municipal y provincial estuvieron presentes en el $77,8 \%$ de los originales, poniendo de manifiesto que la investigación española sobre tabaquismo es fiel a la afirmación de que cada vez es más necesaria la producción de estudios regionales $y$, aún más, locales ${ }^{2}$, queriendo significar que los problemas de salud de una comunidad deben ser resueltos por investigaciones realizadas en la misma comunidad $\mathrm{y}$ no por lo que investiguen otros en otras comunidades.

El predominio de originales realizados desde el ámbito asistencial de la atención primaria $(71,6 \%)$ no debe atribuirse a que la revista con mayor participación en la investigación sobre tabaquismo, Aten Primaria, sea de esa especialidad, ya que ésta aporta sólo el $38,3 \%$ de los originales estudiados, sino más bien, a un mayor abordaje científico del problema del tabaquismo desde este ámbito asistencial.

La población demandante estuvo presente en el $20,8 \%$ de los originales, mientras que en los artículos de Aten Primaria, en el período de 1984 a 1990 , estaba presente en el $30,7 \%{ }^{7}$. La preferencia por este tipo de población puede estar relacionada con las dificultades que plantea diseñar estudios de investigación en poblaciones distintas.

$\Lambda \mathrm{l}$ igual que otros autores, analizando los artículos de revistas, tanto nacionales $^{7,8,10,21,22}$ como internacionales ${ }^{23-26}$, encontramos un alto porcentaje de originales que utilizan diseños observacionales y, especialmente, descriptivos $(67,5 \%)$.

Aunque en un principio puede pensarse que un estudio descriptivo tiene una utilidad baja, esto no es así, porque si su realización se corresponde con una estrategia para planificar estudios analíticos o experimentales, su importancia debe ser considerada elevada. En cualquier caso, este patrón debería cambiar hacia el discño de cstudios analíticos o experimentales, siguiendo la tendencia detectada en algunas revistas 6 , y cuyas 
ventajas han sido ampliamente documentadas ${ }^{18}$.

En este trabajo, podría justificarse el predominio de estudios descriptivos por la escasez de producción científica previa sobre tabaquismo, si bien dicho predominio no ha variado a lo largo de los doce años del estudio, al igual que ocurre con los artículos publicados en 1975 y en 1984 en las revistas Med Clin (Barc) y Rev Clin $E s p^{2}$. Hay que pensar, entonces, en otras causas, como la actitud de los investigadores ante la realización de un trabajo de investigación, preocupados, en muchos casos, por aumentar el número de artículos publicados, más que la calidad de los mismos. Igualmente, habría que relacionar los diseños descriptivos con el predominio observado de estudios sobre patrones de consumo y de las encuestas como fuente de información, en los que no serían necesarios diseños de mayor complejidad.

Diferencias significativas se encontraron en función de la revista de publicación, siendo el porcentaje de originales con diseño descriptivo de alrededor del $50,0 \%$ en las revistas Med Clin (Barc) y Rev Clin Esp, lo que hay que atribuir a los diferentes criterios de selección de artículos por las revistas. En un estudio comparativo de 8 revistas internacionales de reumatología en 1987, se encuentra que el porcentaje de artículos con diseños descriptivos es del $79,5 \%$ en una revista española, $74,6 \%$ en una francesa, $64,2 \%$ y $53,8 \%$ en dos inglesas, $52,7 \%$ en una italiana, $45,2 \%$ en una canadiense, $40,4 \%$ en una estadounidense y $23,4 \%$ en una suiza ${ }^{25}$.

El alto porcentaje de estudios descriptivos en la revista Aten Primaria ha sido justificado por Jiménez y $\mathrm{col}^{7}$ con el hecho de tratarse de una revista joven en la que se publican experiencias de una especialidad también joven, cuyo primcr paso es la descripción de los problemas de salud más frecuentes o de las actividades que se realizan.
Los resultados de este trabajo pueden ser útiles para orientar la investigación sobrc tabaquismo hacia ciertas parcelas prioritarias de la misma, como la prevención, contemplada como actividad fundamental del Sistema Sanitario por la Ley General de Sani$\mathrm{dad}^{27}$, o las motivaciones que inducen a fumar y a la deshabituación, consideradas como prioridades de investigación por el Fondo de Investigaciones Sanitarias ${ }^{28}$. Igualmente, son una llamada de atención a los potenciales autores, comités editoriales y revisores, para que cuiden e identifiquen los aspectos metodológicos de los artículos, mejorando así la calidad de las publicaciones. Sería interesante realizar estudios que analicen la metodología de los artículos publicados sobre otras áreas temáticas de la importancia del tabaquismo.

\section{BIBLIOGRAFÍA}

1. Glänzel W, Schoepflin U. A bibliometric study of reference literature in the sciences and social sciences. Information Processing \& Management 1999; 35: 31-44.

2. Álvarez C, Mur P, Gascón E, Nolasco A, Bolúmar F. La investigación clínica en España: tipos de diseños utilizados. Med Clin (Barc) 1987; 89: 221-3.

3. González JC, Pulido M, Sanz F. Evaluación del uso de procedimientos estadísticos en los artículos originales publicados en Medicina Clínica durante tres décadas (1962-1992). Med Clin (Barc) 1995; 104: 448-52.

4. Mora R, Ascaso C, Scntís J. Uso actual de la estadística en investigación biomédica: una comparación entre revistas de medicina gencral. Med Clin (Barc) 1996; 106: 451-6.

5. Carré MC, Jiménez J, Martín M, Jané F. La estadística en la investigación clínica de medicamentos. Estudio de los artículos originales. Med Clin (Barc) 1996; 106: 611-6.

6. Blasco J, Guerrero JA. Tipos de diseño en la revista Medicina Intensiva. Algo ha cambiado además del dolor. Med Intensiva 1990; 14: 241-4.

7. Jiménez J, Carré MC, Argimón JM. Tipos de estudios publicados en la revista Atención Primaria (1984-1990). Aten Primaria 1993; 12: 23-30. 
8. Beltrán JI, Martínez F. Artículos de Castilla-La Mancha, de interés en atención primaria de salud, publicados en revistas nacionales. Revisión de cinco años (1986-1990). Aten Primaria 1991; 8: 954-60.

9. Jiménę J, Căré MC, Argimón JM. Información sobre los aspectos metodológicos en los artículos de la revista Atención Primaria. Aten Primaria 1994; 14: 1113-7.

10. Álvarez M, López ML, Cueto A. Análisis temático y metodológico de la investigación en atención primaria (1988-1992). Aten Primaria 1996; 18 : 297-303.

11. Ruiz MT, Álvarez C, Bruno ML, Bolúmar F, Pascual E. Investigación en reumatología. Un análisis de la situación española. Med Clin (Barc) 1990; 94: 773-6.

12. Caba $\Gamma$, Echevarría M. Situación actual de la investigación en anestesiología en Andalucía-Extremadura. Estudio comparativo con el resto de España. Rev Esp Anestesiol Reanim 1995; 42: $420-3$.

13. Soto J, Galende I, Sacristán JA. Calidad de los ensayos clínicos publicados en España: valoración a través del análisis de tres revistas durante el período 1985-1991. Med Clin (Barc) 1994; 102: $241-5$.

14. Organización Mundial de la Salud. La lucha contra la epidemia del tabaquismo. Serie de Informes Técnicos núm. 636. Ginebra; 1979.

15. Pinilla R. La estrategia de Salud para Todos en el año 2000 en España. Rev San Hig Pub 1990; 64: 3-7.

16. Sánchez J, Guardiola E. La investigación en dependencia de drogas vista a través de cuatro revistas de la especialidad. Rev Esp Drogodep 1992; 17: 3-15.

17. Gálvez R, Guillén JF, Fernández MA. Concepto y usos de la epidemiología. En: Piédrola $G$, del Rey J, Domínguez M, Cortina P, Gálvez R, Sierra
A et al. Medicina preventiva y salud pública. 90 ed. Barcelona: Masson-Salvat; 1994. p. 79-85.

18. Álvarez C, Bolúmar F, Porta M. Tipos de estudios. Med Clin (Barc) 1987; 89: 296-301.

19. Amat $\mathrm{CB}$, de la Cueva $\Lambda$. Difusión internacional de los trabajos publicados en revistas biomédicas españolas. Un estudio cuantitativo del período 1980-1986. Rev Esp Doc Cient 990; 13: 562-9.

20. López JM, Terrada ML. El consumo de información cientifica nacional y extranjera en las revistas médicas españolas: un nuevo repertorio destinado a su estudio. Med Clin (Barc) 1994; 102: 104-12.

21. Farrús M, de la Fuente, JA, Iglesias M, Borrell C. Revisión de los artículos originales publicados por profesionales de atención primaria en Cataluña. Aten Primaria 1993; 12: 325-32.

22. Soler JM, Solanas JV, Ros A. Investigación en atención primaria en la Comunidad Valenciana. Articulos originales publicados durante 4 años (1990-1993). Aten Primaria 1995; 16: 338-42.

23. Moody LE, Wilson ME, Smyth K, Schartz R, Tittle M, Cott M. Analysis of a decade of nursing practice research: 1977-1986. Nursing Res 1988; 37: 374-9.

24. Geyman JP, Berg AO. The Journal of Family Practice 1974-1988. Window to an evolving academic discipline. J Fam Pract 1989; 28: 301-4.

25. Ruiz MT, Álvarez C, Vela P, Pascual E. Study designs and statistical methods in rheumatological journals: an international comparison. $\mathrm{Br} \mathrm{J}$ Rheumatol 1991; 30: 352-5.

26. Rosenfeld RM. Clinical research in otolaryngology journals. Arch Otolaryngol Head Neck Surg 1991; 117: 164-70.

27. Ley 14/1986, de 25 de abril, General de Sanidad. Boletín Oficial del Estado núm 102, de 29/4/86.

28. Ministerio de Sanidad y Consumo. Fondo de Investigaciones Sanitarias. Convocatoria de acciones 1993. Madrid; 1992. 